\title{
Multi-Level Search Algorithm for Spatial Straightness Error Evaluation
}

\author{
Li HUO ${ }^{a}$, Yuan WANG ${ }^{b}$, Yingchao LI $^{c}$, Yun LIU ${ }^{\mathrm{d}}$, Chen DONG ${ }^{\mathrm{e}}$ and \\ Xuefeng $\mathrm{ZHAO}^{\mathrm{f}}$
}

\author{
Baicheng Ordnance Test Center of China, Baicheng 137001, China \\ a huoli1979@126.com, b wangyuan198110@126.com, ' lychgmlyr1@163.com, \\ diuyun_1988@126.com, e dongchen526@126.com, ${ }^{\mathrm{f}}$ 287106473@qq.com
}

Keywords: Spatial straightness, Error evaluation, Multi-Level search, Spatial straight line fitting, Total least squares

\begin{abstract}
To rapidly and accurately evaluate spatial straightness error, a multi-level search algorithm (MLSA) was proposed. Firstly, the line of measured points was fitted by the total least squares. Then, the measured points were projected on the vertical plane of the fitting line. Finally, the minimum circumscribed circle diameter of the projection points on the plane was calculated by MLSA, the minimum circumscribed circle diameter as the spatial straightness error. The algorithm at the premise of a fixed amount search point of each level, by narrowing the length of the search region to narrow the search step until the required accuracy so far, in order to achieve a refined search. MLSA for evaluating spatial straightness error with higher precision, faster speed, simple and easy to implement, suitable for rapid evaluation of high-precision spatial straightness error.
\end{abstract}

\section{Introduction}

Spatial straightness error is one of the most important parameters in measuring precision hole and shaft parts [1]. According to the National Standard GB/T 11336-2004 [2], there are three methods to evaluate spatial straightness error. They are: linking both ends method, the least square method(LSM) and minimum zone method(MZM).Among them, only MZM conforms to define and its evaluation results are not larger than the other two methods, but it will be hard to solve [3]. The linking both end method is the most simple but the accuracy is also the lowest. LSM is simple and rapid and can meet the requirement of general accuracy so that it is widely used in the production field. Wang [4] on the basis of total least squares method (TLSM) fitting method and space projection is proposed through the lattice method (LM) for spatial straightness error method. This method has good stability and is easy to be programmed. But because the method only on the target region for a search, in the search area is determined to improve the accuracy of the search, only by setting more dense grid to take measures, which will undoubtedly significantly increase the amount of calculation. Similar problems also exist in the roundness error evaluation, and the multi-level search algorithm proposed by the Huo [4] is very effective to achieve the minimum zone method of roundness error evaluation.

In this paper, to rapidly and accurately evaluate spatial straightness error, a multi-level search algorithm (MLSA) is proposed. In the case of a fixed number of points on each search, the search region is reduced continuously by the constant reduction of the search region until the desired accuracy is achieved.

\section{Application TLSM Fitting Spatial Straight line}

The measured points of the hole part are denoted by the Cartesian coordinate $P_{i}\left(x_{i}, y_{i}, z_{i}\right)$ $(i=1,2, \cdots, n ; n \geq 3)$, where $\mathrm{n}$ is the number of measured points.Known from literature [6], in the least squares criterion, the spatial straight line must pass through the data centre $P_{g}\left(x_{g}, y_{g}, z_{g}\right)$. The 
direction vector of a straight line is $s=(u, v, w)$, assuming $u v w \neq 0$, then there is a linear equation such as Eq.1.

$$
\frac{x-x_{g}}{u}=\frac{y-y_{g}}{v}=\frac{z-z_{g}}{w}
$$

for which

$$
\left\{\begin{array}{l}
x_{g}=\sum_{i=1}^{n} x_{i} / n \\
y_{g}=\sum_{i=1}^{n} y_{i} / n \\
z_{g}=\sum_{i=1}^{n} z_{i} / n
\end{array}\right.
$$

Distance from the measured points $P_{i}\left(x_{i}, y_{i}, z_{i}\right)$ to fitting straight line is

$$
d_{i}=\frac{\left|\overrightarrow{P_{i} P_{g}} \times s\right|}{|s|}
$$

After unitize direction vector $s$ (Set $\left.u^{2}+v^{2}+w^{2}=1\right)$, Eq.3 can convert to the form of Eq.4.

$$
d_{i}^{2}=\left(x_{i}-x_{g}\right)^{2}+\left(y_{i}-y_{g}\right)^{2}+\left(z_{i}-z_{g}\right)^{2}-\left[u\left(x_{i}-x_{g}\right)+v\left(y_{i}-y_{g}\right)+w\left(z_{i}-z_{g}\right)\right]^{2}
$$

The geometrical significance of fitting spatial straight line using TLSM is that minimize the squares sum of distance which from all the measured points to object straight line.

$$
\sum_{i=1}^{n} d_{i}^{2}=\min
$$

Combining Eq.4, the Eq.5 is equivalent to Eq.6.

$$
\sum_{i=1}^{n}\left[u\left(x_{i}-x_{g}\right)+v\left(y_{i}-y_{g}\right)+w\left(z_{i}-z_{g}\right)\right]^{2}=\max
$$

According to Lagrangian multipliers method can structure objective function:

$$
f_{\text {TLSM }}=\max \sum_{i=1}^{n}\left[u\left(x_{i}-x_{g}\right)+v\left(y_{i}-y_{g}\right)+w\left(z_{i}-z_{g}\right)\right]^{2}-\lambda\left(u^{2}+v^{2}+w^{2}-1\right)
$$

In the objective function be in the extreme point, should satisfy the condition of Eq.8.

$$
\left\{\begin{array}{l}
\partial f_{\text {TLSM }} / \partial a=0 \\
\partial f_{\text {TLSM }} / \partial b=0 \\
\partial f_{\text {TLSM }} / \partial c=0 \\
\partial f_{\text {TLSM }} / \partial \lambda=0
\end{array}\right.
$$

According to Eq.8 can structure Eq.9.

$$
\boldsymbol{E} \boldsymbol{E}^{\mathrm{T}} \boldsymbol{F}=\lambda \boldsymbol{F}
$$

for which, $\boldsymbol{E}=\left[\begin{array}{ccccc}x_{1}-x_{g} & \cdots & x_{i}-x_{g} & \cdots & x_{n}-x_{g} \\ y_{1}-y_{g} & \cdots & y_{i}-y_{g} & \cdots & y_{n}-y_{g} \\ z_{1}-z_{g} & \cdots & z_{i}-z_{g} & \cdots & z_{n}-z_{g}\end{array}\right], \quad \boldsymbol{F}=\left[\begin{array}{lll}u & v & w\end{array}\right]^{\mathrm{T}}$.

Set $\boldsymbol{G}=\boldsymbol{E} \boldsymbol{E}^{\mathrm{T}} \boldsymbol{G}$ is the real symmetric matrix of the 3 order. The maxima value of the objective function Eq.9 is the maximum eigenvalue $\lambda_{\max }$ of the matrix $\boldsymbol{G}$. Fitting the space vector $(u, v, w)$ of the object straight line, namely corresponding eigenvectors of $\lambda_{\max }$ [7].

\section{Spatial Projection}

By projecting the measured points $P_{i}\left(x_{i}, y_{i}, z_{i}\right)$ to the plane projection which is perpendicular to the least square fitting straight line, the space problem is transformed into a plane problem. The coordinates $\left(X_{i}, Y_{i}\right)$ of the projected points in the plane coordinate system are obtained. 


\section{Step 1 Translation of coordinate system.}

Measuring coordinate system $o-x y z$ through translation get new coordinate system $o^{\prime}-x^{\prime} y^{\prime} z^{\prime}$, the origin $o^{\prime}$ of the new coordinate system and the data center $P_{g}\left(x_{g}, y_{g}, z_{g}\right)$ coincide, as showed in Fig.1(a), then

$$
\left[\begin{array}{lll}
x^{\prime} & y^{\prime} & z^{\prime}
\end{array}\right]^{\mathrm{T}}=\left[\begin{array}{lll}
x & y & z
\end{array}\right]^{\mathrm{T}}-\left[\begin{array}{lll}
x_{g} & y_{g} & z_{g}
\end{array}\right]^{\mathrm{T}}
$$

\section{Step 2 Rotation of coordinate system.}

Suppose the least square fitting straight line $L_{f}$ and $z^{\prime}$ axis angle is $\beta$, least square fitting straight line in the plane $o^{\prime}-x^{\prime} y^{\prime}$ projection line $L_{f}{ }^{\prime}$ and $x^{\prime}$ axis angle is $\alpha$, then $\alpha=\arctan (v / u)$, $\beta=\arccos \left(w / \sqrt{u^{2}+v^{2}+w^{2}}\right)$.

Coordinate system $o^{\prime}-x^{\prime} y^{\prime} z^{\prime}$ around the $z^{\prime}$ axis rotation angle $\alpha$, get the coordinate system $o^{\prime \prime}-x^{\prime \prime} y^{\prime \prime} z^{\prime \prime}$, so that the $x^{\prime \prime}$ axis and the projection line $L_{f}{ }^{\prime}$ coincidence, as shown in Figure 1 (b), then

$$
\left[\begin{array}{l}
x^{\prime \prime} \\
y^{\prime \prime} \\
z^{\prime \prime}
\end{array}\right]=\left[\begin{array}{ccc}
\cos \alpha & \sin \alpha & 0 \\
-\sin \alpha & \cos \alpha & 0 \\
0 & 0 & 1
\end{array}\right]\left[\begin{array}{l}
x^{\prime} \\
y^{\prime} \\
z^{\prime}
\end{array}\right]
$$

Coordinate system $o^{\prime \prime}-x^{\prime \prime} y^{\prime \prime} z^{\prime \prime}$ around the $y^{\prime \prime}$ axis rotation angle $\beta$, get the coordinate system $O-X Y Z$, so that the $Z$ axis and the line $L_{f}$ coincidence, as shown in Figure 1 (c), then

$$
\left[\begin{array}{l}
X \\
Y \\
Z
\end{array}\right]=\left[\begin{array}{ccc}
\cos \beta & 0 & -\sin \beta \\
0 & 1 & 0 \\
\sin \beta & 0 & \cos \beta
\end{array}\right]\left[\begin{array}{l}
x^{\prime \prime} \\
y^{\prime \prime} \\
z^{\prime \prime}
\end{array}\right]
$$

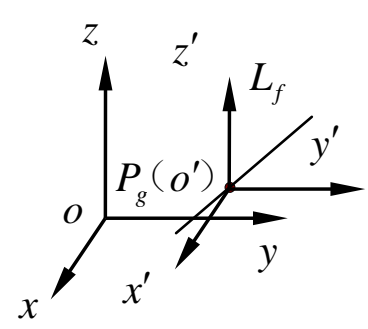

(a)

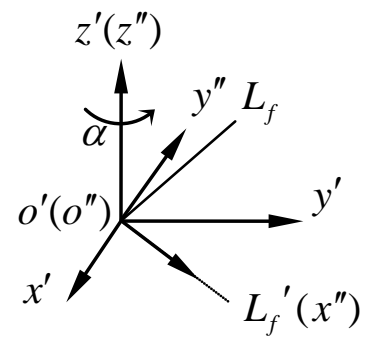

(b)

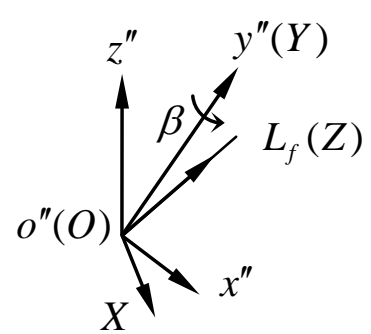

(c)

Fig.1 Transformation schematic diagram of coordinate system

\section{Step 3 Coordinate conversion.}

Combined Eq.10, Eq.11 and Eq.12 can be formed as Eq.13.

$$
\left[\begin{array}{l}
X \\
Y \\
Z
\end{array}\right]=\left[\begin{array}{ccc}
\cos \beta & 0 & -\sin \beta \\
0 & 1 & 0 \\
\sin \beta & 0 & \cos \beta
\end{array}\right]\left[\begin{array}{ccc}
\cos \alpha & \sin \alpha & 0 \\
-\sin \alpha & \cos \alpha & 0 \\
0 & 0 & 1
\end{array}\right]\left[\begin{array}{l}
x-x_{g} \\
y-y_{g} \\
z-z_{g}
\end{array}\right]
$$

The measured points coordinates $P_{i}\left(x_{i}, y_{i}, z_{i}\right)$ into Eq.13 calculated the specified coordinates projection points $\left(X_{i}, Y_{i}\right)$ on the plane.

\section{Multi-Level Search Algorithm}

\subsection{Setting of the Initial Level Search Region}

In order to facilitate the calculation of the computer, each level of the search region is set as a square. This paper take the data center $G_{0}\left(X_{0}, Y_{0}\right)$ of all the projection points as the center $O_{1}\left(a_{1}, b_{1}\right)$ of the initial level search region $(m=1)$; with $O_{1}$ as the center, all inclusive smallest circle projection points and the diameter of $D_{0}$ is the initial level of the search region length $d_{1}$. Then there is $a_{1}=X_{0}$, 
$b_{1}=Y_{0}, d_{1}=D_{0}$. According to Eq.14 to calculate the coordinates of the projection point data center $G_{0}\left(X_{0}, Y_{0}\right)$.

$$
\left\{\begin{array}{l}
X_{0}=\sum_{i=1}^{n} X_{i} / n \\
Y_{0}=\sum_{i=1}^{n} Y_{i} / n
\end{array}\right.
$$

According to Eq.15 to calculate the center of $O_{1}$ search region as the center of the circle of the minimum diameter of $D_{0}$.

\subsection{Division of Mesh Points}

$$
D_{0}=2 \max \left\{\sqrt{\left(X_{i}-X_{0}\right)^{2}+\left(Y_{i}-Y_{0}\right)^{2}}\right\}
$$

Divide every side of each level search region into $l$ equal parts ( $l$ is an even number), then each side has $(l+1)$ equal division points, and every two links constitute $(l+1) \times(l+1)$ mesh points.

Set the $m(m \geq 1)$ level search, the search region of the center for $O_{m}\left(a_{m}, b_{m}\right)$, the search region length is $d_{m}$, the mesh size is $g_{m}=d_{m} / l$.According to Eq. 16 work out all coordinates $\left(x_{m j}, y_{m k}\right)$ of mesh point when searching the $\mathrm{m}$ level.

In the equation:

$$
\left\{\begin{array}{l}
x_{m j}=a_{m}+j g_{m} \\
y_{m k}=b_{m}+k g_{m}
\end{array}\right.
$$

$j=-l / 2,-l / 2+1, \cdots,-2,-1,0,1,2, \cdots, l / 2-1, l / 2 ; \quad k=-l / 2,-l / 2+1, \cdots,-2,-1,0,1,2, \cdots, l / 2-1, l / 2$.

\subsection{Target Mesh Point Search of Each Level}

According to Eq. 17 is calculated in each grid point $\left(x_{m j}, y_{m k}\right)$ as the center of the minimum circle diameter $D\left(x_{m j}, y_{m k}\right)$, when search m-th level.

$$
D\left(x_{m j}, y_{m k}\right)=2 \max \left\{\sqrt{\left(x_{i}-x_{m j}\right)^{2}+\left(y_{i}-y_{m k}\right)^{2}}\right\}
$$

Satisfy the condition of Eq.18 namely is the minimum inclusive circle diameter $D_{m}$ which gets by searching the m-th level. The corresponding mesh point is the point of the target, and write down as $G_{m}\left(X_{m}, Y_{m}\right)$.

\subsection{Ascertainment of New Search Region}

$$
D_{m}=\min \left\{D\left(x_{m j}, y_{m k}\right)\right\}
$$

When $m$ is greater than 2, the center of the search region is the target mesh point of the $(m-1)$ level, the search region side is $d_{m}$ according to Eq.19 calculation.

$$
d_{m}=2 \max \left\{\left|a_{m}-a_{m-1}\right|,\left|b_{m}-b_{m-1}\right|, g_{m-1}\right\}
$$

The algorithmic program flow diagram is shown in Fig.2. 


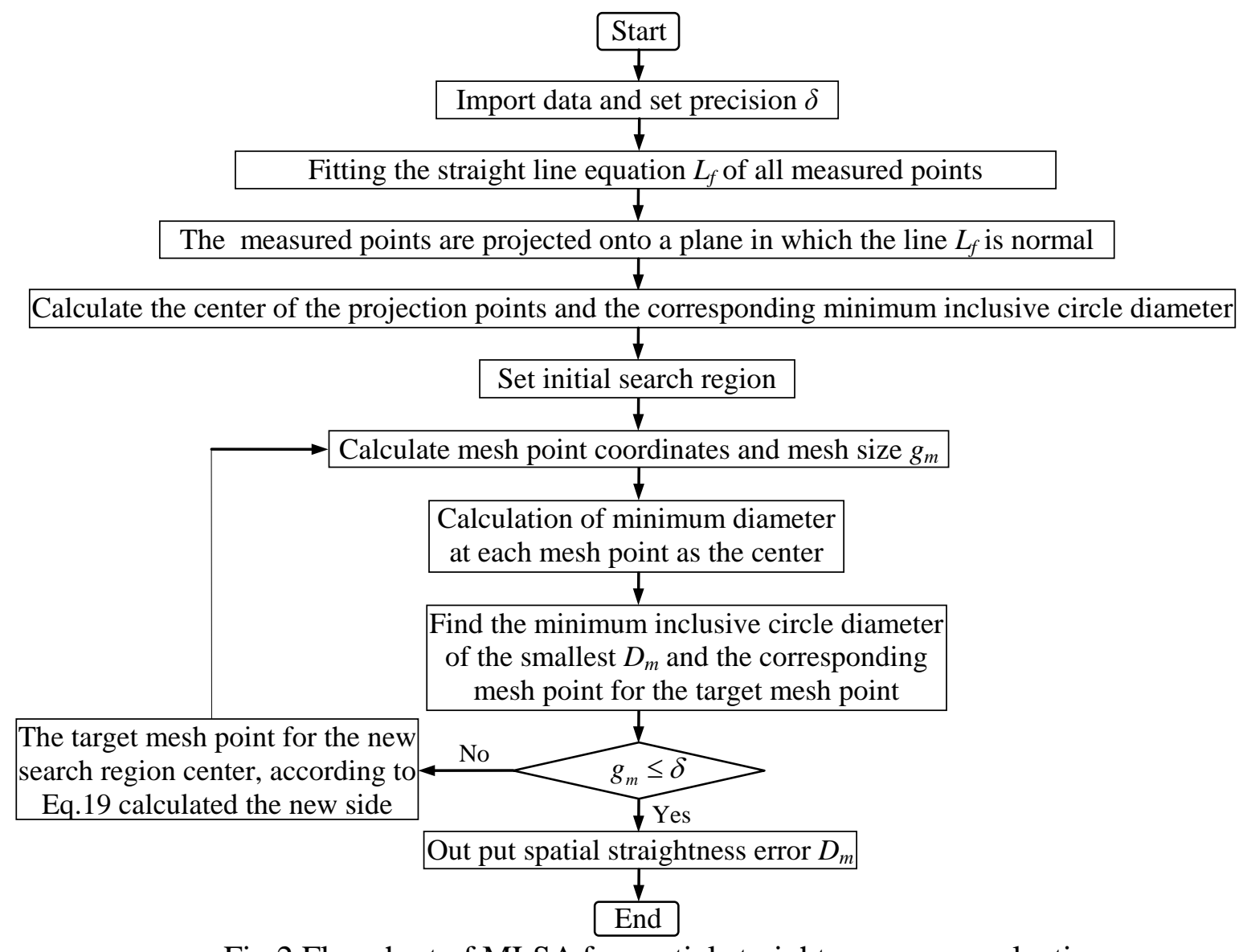

Fig.2 Flowchart of MLSA for spatial straightness error evaluation.

\section{Experimental Results}

Using the dataset of literature [3], the evaluation results of various algorithms are listed in table 1. The present algorithm has set up 100 level mesh search, and the mesh points of each level are $101 \times 101$.

Table 1 Evaluation results

\begin{tabular}{cc}
\hline Algorithm & $\begin{array}{c}\text { Spatial straightness error } \\
/ \mu \mathrm{m}\end{array}$ \\
\hline Genetic algorithm $^{[3]}$ & 26.0000 \\
Particle swarm optimization $^{[8]}$ & 24.9320 \\
Successive quadratic programming $^{[9]}$ & 15.1360 \\
3DLSA $^{[10]}$ & 13.5000 \\
LSABC $^{[11]}$ & 11.8475 \\
Improvement LSM algorithm $^{[12]}$ & 11.8000 \\
LM $^{[4]}$ & 9.9764 \\
Present algorithm & 9.9758 \\
\hline
\end{tabular}

From table 1, compared with the evaluation results of the comparison algorithms, the result of the present algorithm is the best, only the result of LM algorithm of the literature [4] is the closest to the present algorithm. Different algorithms are programmed to run on the same computer. The result of $\mathrm{LM}$ algorithm is $9.97591 \mu \mathrm{m}$, takes time $15.25 \mathrm{~s}$; the result of the present algorithm is $9.97577 \mu \mathrm{m}$, takes time $1.94 \mathrm{~s}$. It also shows that the algorithm is more accurate and faster. 


\section{Conclusions}

In this paper, we proposed the Multi-Level Search Algorithm (MLSA) which for evaluating the spatial straightness error with higher precision and faster speed. Moreover, in contrast to algorithms in literature, the principle of the algorithm is simple and is easy to be achieved by computer.

\section{References}

[1] J.LUO, Q.WANG. A method for axis straightness error evaluation based on improved artificial bee colony algorithm. The International Journal of Advanced Manufacturing Technology.Vol.71(2014),p.1501-1509.

[2] Standardization Administration of the People's Republic of China. GB/T 11336-2004 Measurement of departures from straightness. Standards Press of China, Beijing, 2004.

[3] P.LIAO, S.Y.YU. A method of calculating 3-D line error using genetic algorithm. Journal of Central South University Technology.Vol.29(1998) No.6,p.586-588.

[4] B.J.WANG, J.P.ZHAO, C.J.WANG. Spatial straightness error evaluation based on three-dimensional least squares method. Journal of Beijing University of Aeronautics and Astronautics.Vol.40(2014) No.10,p.1477-1480.

[5] L.HUO, Y.WANG, C.Y.ZHAO, et al. Multi-level search algorithm for roundness error evaluation by the minimum zone circle method.doi:10.2991/ifmeita-16.2016.57.

[6] M.F.DU. Fitting of space straight line. Journal of Beijing Institute of Printing.Vol.4(1996) No.2,p.27-31.

[7] G.R.PAN, H.TANG. Application of eigen decomposition and selecting weight iteration in spatial line fitting. Journal of Southeast University: Natural Science Edition.Vol.43(2013) Sup 2,p.250-255.

[8] J.MAO, Y.L.CAO. Evaluation method for spatial straightness errors based on particle swarm optimization. Journal of Engineering Design.Vol.13(2006) No.5,p.291-294.

[9] W.L.YUE, Y.WU. Evaluation of spatial straightness errors based on multi-target optimization. Optics and Precision Engineering.Vol.16(2008) No.8,p.1423-1428.

[10] Z.X.HU, X.J.YANG, X.Z.JIN. Research on 3DLSA for evaluating spatial straightness errors. China Mechanical Engineering.Vol.21(2010) No.3,p.326-329.

[11] Z.X.HU, X.J.YANG, F.L.WANG. Research on LSABC algorithm for evaluation of spatial straightness error. Journal of Engineering Design.Vol.15(2008) No.3,p.187-190,212.

[12] Z.X.HU, X.J.YANG, X.Z.JIN. Analysis and improvement of the LSM algorithm for assessing spatial straightness error. Journal of Hunan University: Natural Sciences.Vol.37(2010) No.2,p.27-31. 